\title{
Dynamique Des Eléments Nutritifs Et Du Phytoplancton Dans Le Barrage Béni Haroun Dans l'Est Algérien
}

\author{
Kherief Nacereddine $S$. \\ Djeddi $\boldsymbol{H}$. \\ Benayache N. $Y$. \\ Afri-Mehennaoui $F$. $Z$.
}

Laboratoire de Biologie et Environnement Faculté des Sciences de la Nature et de la Vie Université Frères Mentouri Constantine 1. Algérie

Doi: 10.19044/esj.2018.v14n12p111 URL:http://dx.doi.org/10.19044/esj.2018.v14n12p111

\begin{abstract}
In order to monitor the water quality of the Beni Haroun dam, used for the drinking water production, our research work aims to provide new scientific data on the phytoplankton population of the dam. This contribution is a qualitative and quantitative investigation of this important link in the ecosystem equilibrium. To our knowledge, this study has never been the subject of a previous one, despite many works already done on this dam. Water samples were collected monthly from January to December 2015 at five stations, spread over Beni Haroun dam. The exploitation of a database, relating to fourteen physicochemical and biotic variables, made it possible to determine: the spatiotemporal evolution of each variable, the correlations between variables, the annual variability of the algal biomass, the diversity and phytoplankton dominance.
\end{abstract}

Keywords: Beni Haroun Dam, Water quality, Nutrients, Phytoplankton

\section{Résumé}

Dans le but de la surveillance de la qualité des eaux du barrage Béni Haroun utilisées pour la production d'eau potable et d'irrigation, notre travail a comme objectif de recherche et d'apporter de nouvelles données scientifiques sur le peuplement phytoplanctonique du barrage. Cette contribution est une investigation qualitative et quantitative de cet important maillon de l'équilibre de l'écosystème qui, à notre connaissance, n'a jamais fait l'objet d'une étude antérieure malgré la multitude de travaux déjà réalisés sur ce barrage. Des échantillons d'eau ont été prélevés mensuellement de 
janvier à décembre 2015 au niveau de cinq stations, réparties sur le Béni Haroun. L'exploitation d'une base de données, relative à quatorze variables physico-chimiques et biotiques, a permis de déterminer: l'évolution spatiotemporelle de chaque variable, les corrélations entre variables, la variabilité annuelle de la biomasse algale, de la diversité et de la dominance phytoplanctonique.

Mots clés : Barrage Béni Haroun, Qualité de l'eau, Eléments nutritifs, Phytoplancton

\section{Introduction}

La pollution des écosystèmes aquatiques est actuellement l'un des problèmes les plus préoccupants du siècle, que de nombreux pays dans le monde s'attachent à résoudre comme un véritable défi. En effet, l'eau est la ressource naturelle, à la fois la plus indispensable à toute forme de vie et la plus menacée vu l'extension illimitée des unités industrielles à proximité des lacs et des cours d'eau. L'Algérie est l'un des pays où la disponibilité de l'eau est limitée alors que les besoins ne cessent d'augmenter. Cette demande croissante est due à l'explosion démographique, aux développements industriels et agricoles ainsi qu'aux fréquents épisodes de sécheresse. Pour faire face à cette pénurie, la construction des barrages semble être la solution la plus adaptée pour répondre aux différents besoins (Fouad, 2014; BouzidLagha et al., 2011). L'utilité de ces eaux réside essentiellement dans les usages domestiques, agricoles et industriels qu'elles assurent; aussi convient-t-il de suivre leur état de pollution. Les pollutions les plus graves à ce jour qui touchent le bassin de Béni-Haroun, sont celles dues aux activités humaines, à savoir les pratiques agricoles, industrielles, rejets domestiques diffus et, dans une moindre mesure, aux retombées atmosphériques. Ces polluants sont entraînés par les eaux de ruissellement vers le réservoir. L'une des conséquences de cette situation est l'enrichissement de l'eau en nutriments, particulièrement le phosphore et l'azote, indicateurs d'un état avancé d'eutrophisation des eaux (El Ghachtoul et al., 2005). Le terme " eutrophisation "s'est alors généralisé pour exprimer le phénomène de développement anarchique des espèces algales au niveau des couches superficielles des retenues de barrage. Ce développement entraîne une dégradation de la qualité des eaux, en causant des préjudices environnementaux, sociaux et économiques (Abdallaoui, 1998). La prolifération phytoplanctonique est contrôlée essentiellement par des variables physico-chimiques (El Hachimi, 2012). Dépendant des conditions du milieu, ces organismes microscopiques vivent dans la zone euphotique, soit à la strate supérieure des océans, mers, lacs, réservoirs ou étangs d'eau douce (Findlay et al., 1990). La qualité sanitaire de l'eau potable devient alors un objectif 
majeur (Cambon, 2008). Le phytoplancton est un bon indicateur de l'état trophique des eaux. La présente étude représente la première contribution réalisée sur ce premier maillon de la chaîne trophique qui, à notre connaissance, n'a jamais fait objet d'une étude antérieure au niveau du barrage Béni-Haroun malgré la multitude de travaux déjà réalisés sur ce réservoir. Dans ce contexte, et lors de ce travail, nous aurons comme objectif d'examiner la dynamique d'un ensemble de paramètres biotiques et abiotiques dans le temps et dans l'espace et leur effet sur le développement de la population micro-algale.

\section{Présentation de la zone d'étude}

Le barrage de Béni Haroun ( Fig.1), est implanté dans la commune de Hamala, daïra de Grarem Gouga (DTM, 2008). Distant de moins de $15 \mathrm{~km}$ de la wilaya de Mila, il est situé à une quarantaine de $\mathrm{km}$ au Nord de Constantine. La digue est élevée sur oued El Kébir en aval de la confluence des oueds Endja et Rhumel, principaux cours d'eau alimentant le réservoir. Destiné entre autres à l'alimentation en eau potable, le barrage béni Haroun est la plus grande infrastructure hydraulique en Algérie. Il est érigé dans la région bien arrosée du Tell oriental, au Sud de laquelle se situent de grands centres urbains (Constantine, Batna, Khenchela, Oum el-Bouaghi et Mila) et de vastes terres irrigables (hautes plaines semi-arides) qui ne peuvent être satisfaites par les ressources locales. Au plan morpho-géologique, le barrage et son système de transfert sont localisés dans une zone complexe qui engendre des contraintes d'implantation des ouvrages hydrauliques (Mebarki et al., 2008). Sa mise en eau a commencé en 2004 (Benfetta, 2016). La région est caractérisée par son appartenance au climat méditerranéen de type subhumide à semi-aride. L'année 2015 est caractérisée par des précipitations irrégulières, et marquée par deux saisons bien distinctes (Fig.2) : une saison des pluies courte (janviermars) et une longue saison sèche (avril-décembre).

\section{Sites de prélèvement}

Le choix des 5 stations de prélèvement est basé sur leur appartenance au réservoir, leur accessibilité et les sources potentielles de pollutions. Elles sont localisées géographiquement comme suit (coordonnées GPS et altitude) (Tab.1 et Fig. 1).

Tableau 1: Localisation des 5 stations de prélèvement

\begin{tabular}{ccccc}
\hline Stations & Code & Localisation & GPS & Altitude \\
\hline \multirow{2}{*}{ Station 1 } & \multirow{2}{*}{ St1 } & $\begin{array}{c}\text { proche de l'oued Endja, située en } \\
\text { aval de mechtet Mellel }\end{array}$ & $\begin{array}{l}\text { N: } 36^{\circ} 29^{\prime} 55,06^{\prime \prime} \\
\text { E: } 06^{\circ} 07^{\prime} 14,74^{\prime \prime}\end{array}$ & \multirow{2}{*}{$199 \mathrm{~m}$} \\
\hline \multirow{2}{*}{ Station 2 } & \multirow{2}{*}{ St2 } & $\begin{array}{c}\text { située en aval de l'agglomération } \\
\text { de Djellama }\end{array}$ & $\begin{array}{l}\text { N:36³ } 30^{\prime} 23,05^{\prime \prime} \\
\text { E: } 06^{\circ} 11^{\prime} 51,79^{\prime \prime}\end{array}$ & \multirow{2}{*}{$203 \mathrm{~m}$} \\
\hline \multirow{2}{*}{ Station 3 } & \multirow{2}{*}{ St3 } & $\begin{array}{c}\text { proche d'oued Metllili en aval } \\
\text { de mechtet Metllili }\end{array}$ & $\begin{array}{l}\text { N: } 36^{\circ} 29^{\prime} 26,8^{\prime \prime} \\
\text { E: } 06^{\circ} 17^{\prime} 39,16^{\prime \prime}\end{array}$ & \multirow{2}{*}{$195 \mathrm{~m}$} \\
\hline
\end{tabular}




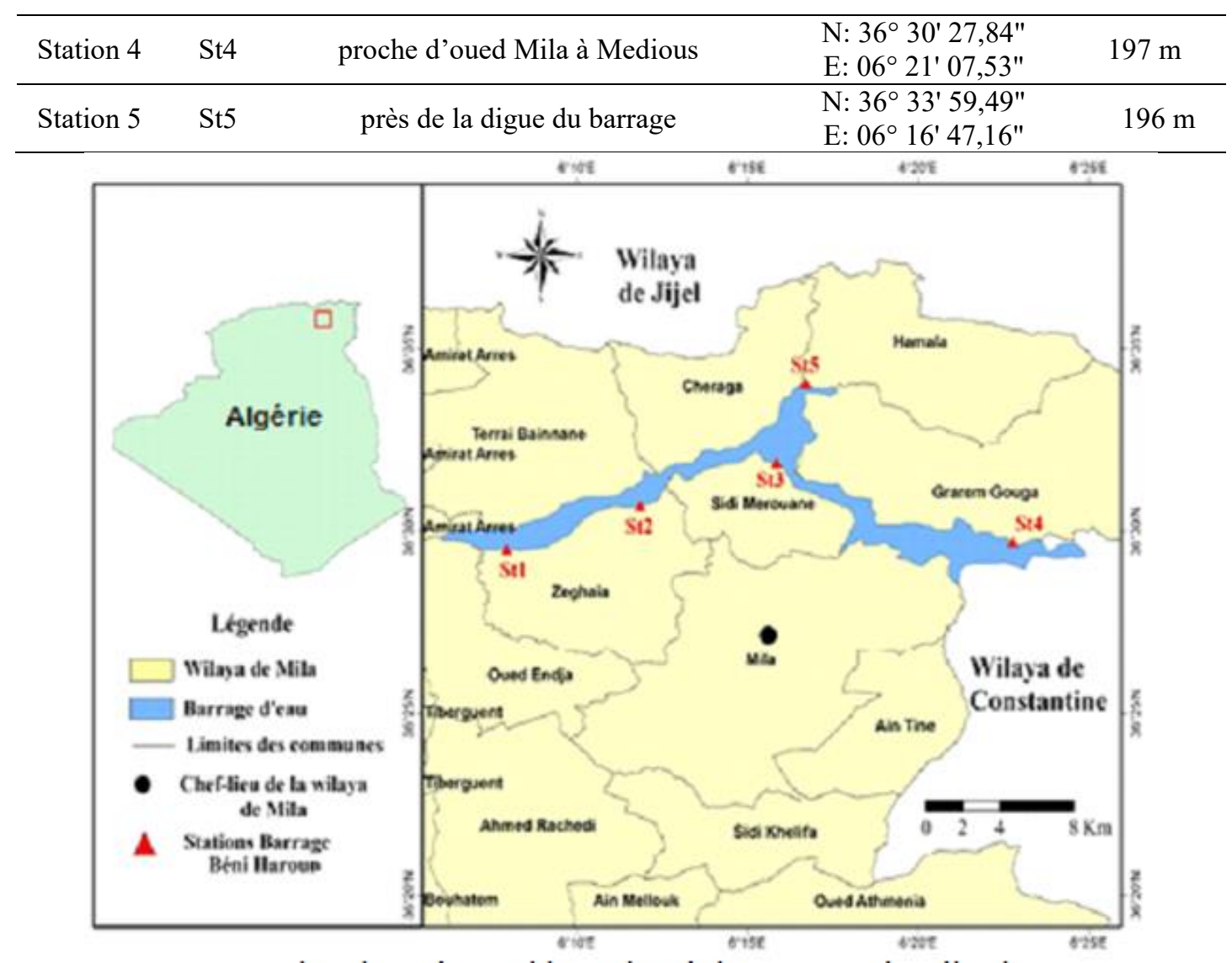

Figure 1: Situation géographique de Béni Haroun et localisation des points de prélèvement

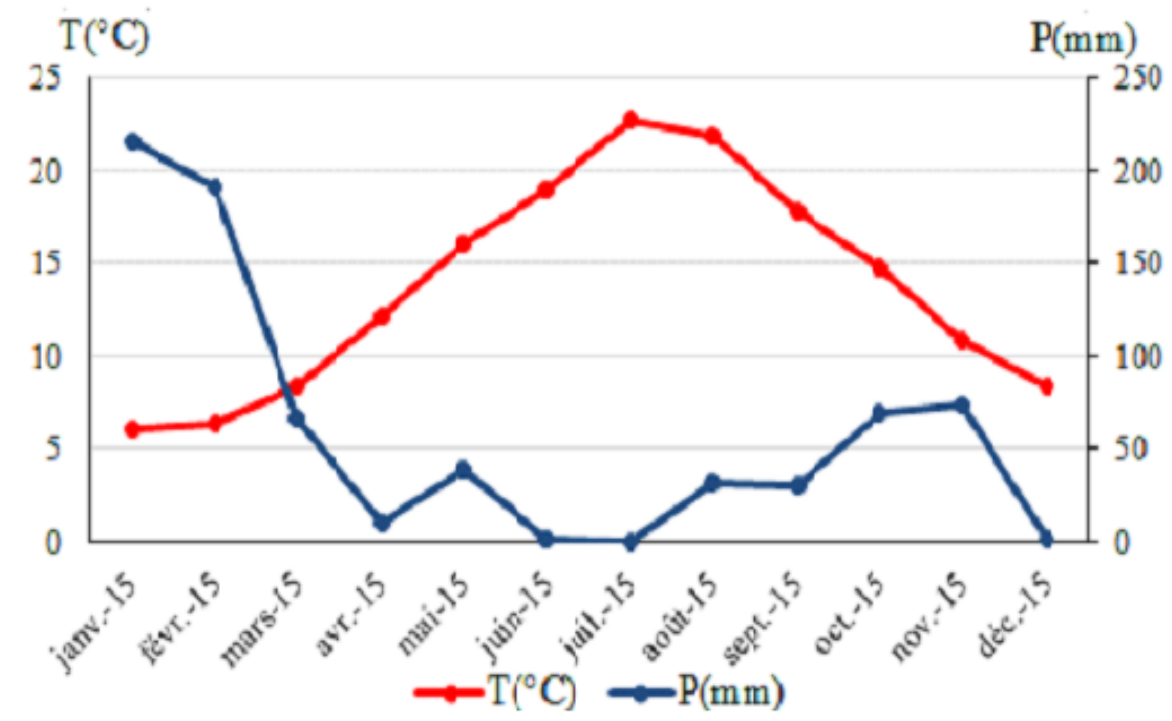

Figure 2: Diagramme ombrothermique de la région d'étude 


\section{Matériel et méthodes}

L'évaluation de la qualité des eaux nécessite une connaissance, aussi complète que possible, des caractéristiques physico-chimiques et biologiques des eaux. A cet effet, treize (13) variables ( $\mathrm{T} \circ, \mathrm{pH}, \mathrm{CE}, \mathrm{O}_{2}$ dissous, $\mathrm{Ca}^{2+}, \mathrm{Mg}^{2+}$, $\mathrm{Na}^{+}, \mathrm{K}^{+}, \mathrm{Cl}^{-}, \mathrm{SO}_{4}^{-2}, \mathrm{NO}_{2}^{-}, \mathrm{NO}_{3}^{-}, \mathrm{PO}_{4}^{-2}$ ) ainsi que des variables biotiques ont été retenues. Durant l'année 2015, douze prélèvements mensuels ont été effectués en surface, au niveau de cinq stations situées sur le pourtour du barrage (Fig.1). Les paramètres physico-chimiques: Potentiel hydrogène, conductivité, température et oxygène dissous, sont mesurés in situ moyennant respectivement un $\mathrm{pH}$-mètre, un conductimètre, un thermomètre et un oxymètre d'un multi-paramètre de mesure WTW (Multi 3420 SET G). Les teneurs des éléments nutritifs sont déterminées selon les méthodes décrites dans l'analyse de l'eau (Rodier, 2009). Les méthodes utilisées sont volumétriques, colorimétriques ou spectrophotométriques moléculaires. Le phytoplancton est étudié à partir d'échantillons d'eau brute prélevés à la surface de la zone euphotique. Les analyses quantitatives du phytoplancton ont été réalisées sur un litre d'eau prélevé à l'aide d'une bouteille ordinaire de cette capacité. L'aspect qualitatif du phytoplancton a été déterminé selon des traits verticaux et horizontaux à l'aide d'un filet à plancton de $20 \mu \mathrm{m}$ de maillage. L'examen qualitatif et quantitatif est réalisé à l'aide d'un microscope de type Leica, après fixation au lugol et concentration des organismes par sédimentation. Les cellules, les colonies et les filaments, ont été comptés en triplicats à la cellule de Thoma, au microscope optique (x 400). L'identification a nécessité des clefs de détermination spécialisées dont celles de Bourrelly (1966, 1968, 1970), Drebes (1976), Germain (1981), Balech (1988), Krammer Lange-Bertalot (1986, 1988, 1991a, 1991b) et Lavoie et al (2008). Les analyses statistiques ont été réalisées à l'aide du logiciel (SPSS19).

\section{Résultats et interprétation \\ Paramètres physico-chimiques}

Les paramètres physico-chimiques mesurés varient légèrement dans l'espace et significativement dans le temps durant la période d'étude (Fig.3). La température de l'eau varie régulièrement avec celle de l'air en présentant des fluctuations thermiques variantes selon la saison, atteignant des valeurs minimales en hiver $\left(12^{\circ} \mathrm{C}\right.$ le matin) et maximales en été $\left(34^{\circ} \mathrm{C}\right.$ à midi pouvant être plus élevées l'après-midi). Ces fluctuations thermiques ont un impact direct sur le développement des populations algales et ce par leur grande influence sur l'activité photosynthétique. El Hachimi (2012) et Smruti (2016) ont aussi observé l'influence de la lumière et de la température sur la croissance et la diversité du phytoplancton. Ce sont les principaux facteurs limitants de la croissance algale. Le pH de l'eau demeure plutôt alcalin et varie 
globalement entre 7 et 9,1. Les valeurs les plus alcalines sont attribuées à la saison estivale. Cette alcalinité favorise la productivité du phytoplancton (Arrignon, 1976; Afri et al., 1983; Bouzid-Lagha et al., 2012). Les valeurs de la conductivité indiquent une minéralisation très élevée dépassant 1000 $\mu \mathrm{S} / \mathrm{cm}$. Un pic de $3800 \mu \mathrm{S} / \mathrm{cm}$ est enregistré en février à la station 1 révélant, selon Rodier (2009), une minéralisation excessive. Cette minéralisation à tendance à s'accroitre suite aux rejets polluants d'origine domestique et agricole, généralement observés aux alentours des stations de prélèvement à l'exception de la station 5 (digue du barrage) sujette au brassage des eaux. Les valeurs de l'oxygène dissous varient entre 3,2 et $19,9 \mathrm{mg} / \mathrm{l}$, marquants une sursaturation (262\%) en avril à la station 5. Les concentrations plus élevées sont paradoxalement enregistrées pendant la saison printanière et ce en raison de l'importante activité photosynthétique qui débute au mois d'avril.

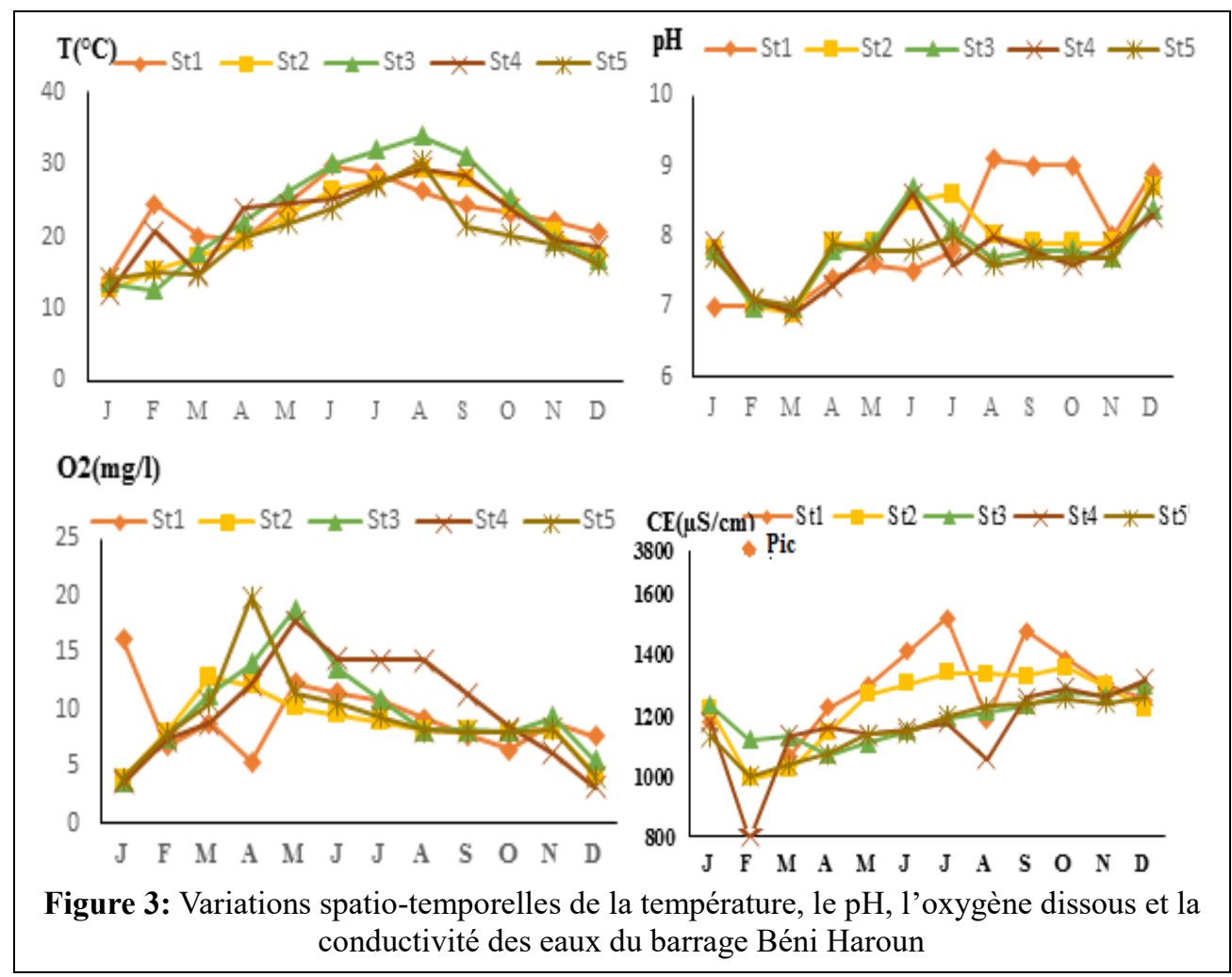

\section{Paramètres nutritifs}

Les deux formes d'azote analysées, nitrates et nitrites (Fig. 4), varient temporellement de 5 à $13,53 \mathrm{mg} / \mathrm{l}$ pour les premiers et de 0,02 à $1,2 \mathrm{mg} / 1$ pour les seconds. Les teneurs les plus élevées sont enregistrées en hiver et à la fin de la saison automnale dans les stations les plus exposées aux apports de lessivage et d'activités anthropiques, en partie dues à l'utilisation intensive 
d'engrais azotés. Ils se trouvent essentiellement dans les engrais et constituent la source majeure de matières azotées des plantes. Les deux tiers de la pollution par les nitrates sont causés par les cultures et l'élevage intensif (Derradji et al., 2007) auxquels s'ajoutent ceux de la minéralisation ultime des autres formes azotées (Norganique, $\mathrm{NH}_{4}{ }^{+}, \mathrm{NO}_{2}{ }^{-}$) rejetées en amont. Cependant les teneurs des $\mathrm{NO}_{3}{ }^{-}$relevées, inférieures à la valeur guide $25 \mathrm{mg} / 1$ (Rodier, 2009) dans le barrage Béni-Haroun, lui confèrent une qualité normale répondant à la qualité des eaux douces superficielles destinées à la production d'eau potable. Les phosphates fluctuent entre 0,5 et $1,07 \mathrm{mg} / \mathrm{l}$, valeur supérieure à $0,7 \mathrm{mg} / 1$ nécessitant un traitement poussé lorsque l'eau est destinée à la consommation humaine (Rodier, 2009). Selon les normes admis par l'ABH, ces valeurs non négligeables sont essentiellement la conséquence des rejets domestiques et des engrais phosphatés utilisés en agriculture sur les berges et le bassin versant du barrage. Les concentrations en sulfates varient entre 109 et $675 \mathrm{mg} / \mathrm{l}$. La présence de sulfates est avant tout liée à la nature géologique de la roche mère et des terrains de la région. Les teneurs les plus élevées sont enregistrées pendant la saison estivale ce qui peut être la conséquence du phénomène d'évaporation qui aura tendance à concentrer les sels. Une contamination directe de l'eau du barrage par des rejets domestiques surtout riches en détergents ou par l'utilisation des pesticides en agriculture (Harrat et al., 2010) n'est pas non plus à exclure. La teneur guide des sulfates pour les eaux superficielles destinées à l'alimentation en eau des populations est $400 \mathrm{mg} / \mathrm{l}$ (Joradp, 2011) alors qu'elle est de 250 mg/l selon Rodier (2009). Les teneurs des chlorures révèlent une variation spatio-temporelle entre 73 et $218 \mathrm{mg} / 1$ avec un pic enregistré en février $(718 \mathrm{mg} / 1)$ comme rapporté pour la conductivité, dû aux rejets véhiculés par l'oued Endja qui reçoit des effluents urbains non épurés. L'impact est décelable sur plusieurs paramètres comme la $\mathrm{CE}, \mathrm{NO}_{3}{ }^{-}, \mathrm{NO}_{2}^{-}, \mathrm{PO}_{4}{ }^{-2}$. Cependant les teneurs en chlorures restent inférieures à la valeur maximale de $600 \mathrm{mg} / \mathrm{l}$ retenue pour les eaux superficielles destinées à l'alimentation en eau des populations (Joradp, 2011) et $200 \mathrm{mg} / \mathrm{l}$ selon Rodier (2009). Les résultats des dosages de la dureté magnésienne et calcique sont respectivement de 60,12 et $136,27 \mathrm{mg} / 1$ et de 14,58 et $97.69 \mathrm{mg} / \mathrm{l}$, ces valeurs sont inférieures aux normes OMS qui sont respectivement $200 \mathrm{mg} / \mathrm{l}$ pour le calcium et $150 \mathrm{mg} / 1$ pour le magnésium. Les valeurs du sodium fluctuent entre 71 et $155 \mathrm{mg} / 1$ celles du potassium de 2,1 à 9,7 mg/l. Les valeurs du sodium, dépassent légèrement celle admise par 1'OMS qui est 150 mg/l (Harrat, 2006). Selon, les normes des eaux de surface (ABH, 1999) ; la qualité des eaux se balance spatio-temporellement entre bonne à passable. Il faut souligner la minéralisation souvent excessivement des eaux du bassin versant Kébir-Rhumel due essentiellement à la nature géologique des terrains drainés. 


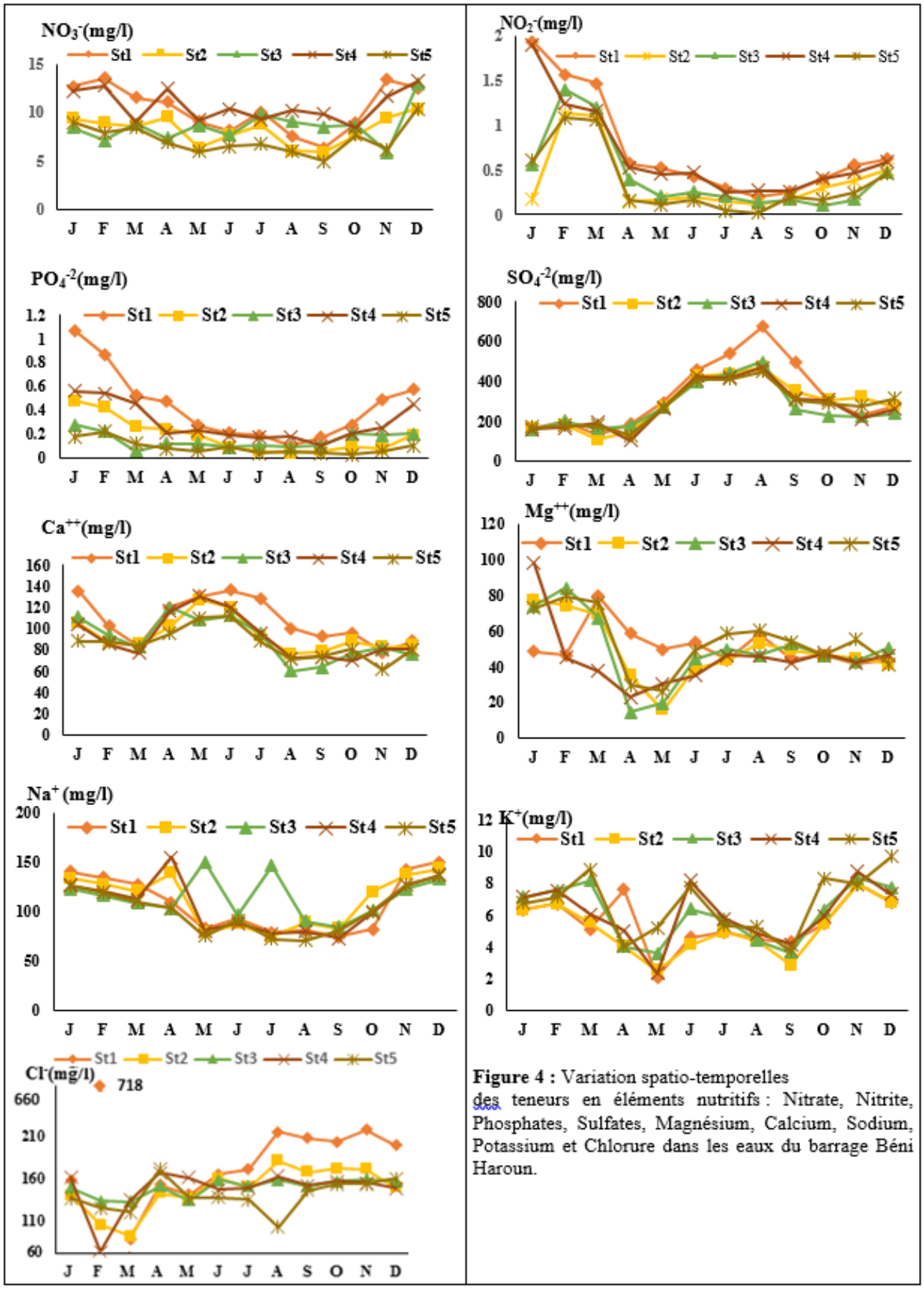

\section{Paramètres biotiques}

La diversité et la densité du phytoplancton sont utilisées comme indicateurs de la qualité de l'eau dans les lacs et les réservoirs (Badsil et al., 
2012). La composition en pourcentage des différents groupes du phytoplancton identifiés, illustrée par la figure 5, indique que la station 1 est caractérisée par l'abondance des Cyanophyceae et Chlorophyceae qui représentent à deux $58 \%$ du peuplement phytoplanctonique. On note la présence non négligeable des Euglenophyceae (15\%) et les Bascillariophyceae $(13 \%)$ puis les Dinophyceae avec une contribution de $8 \%$ et à la dernière position les Mediophyceae (6\%).

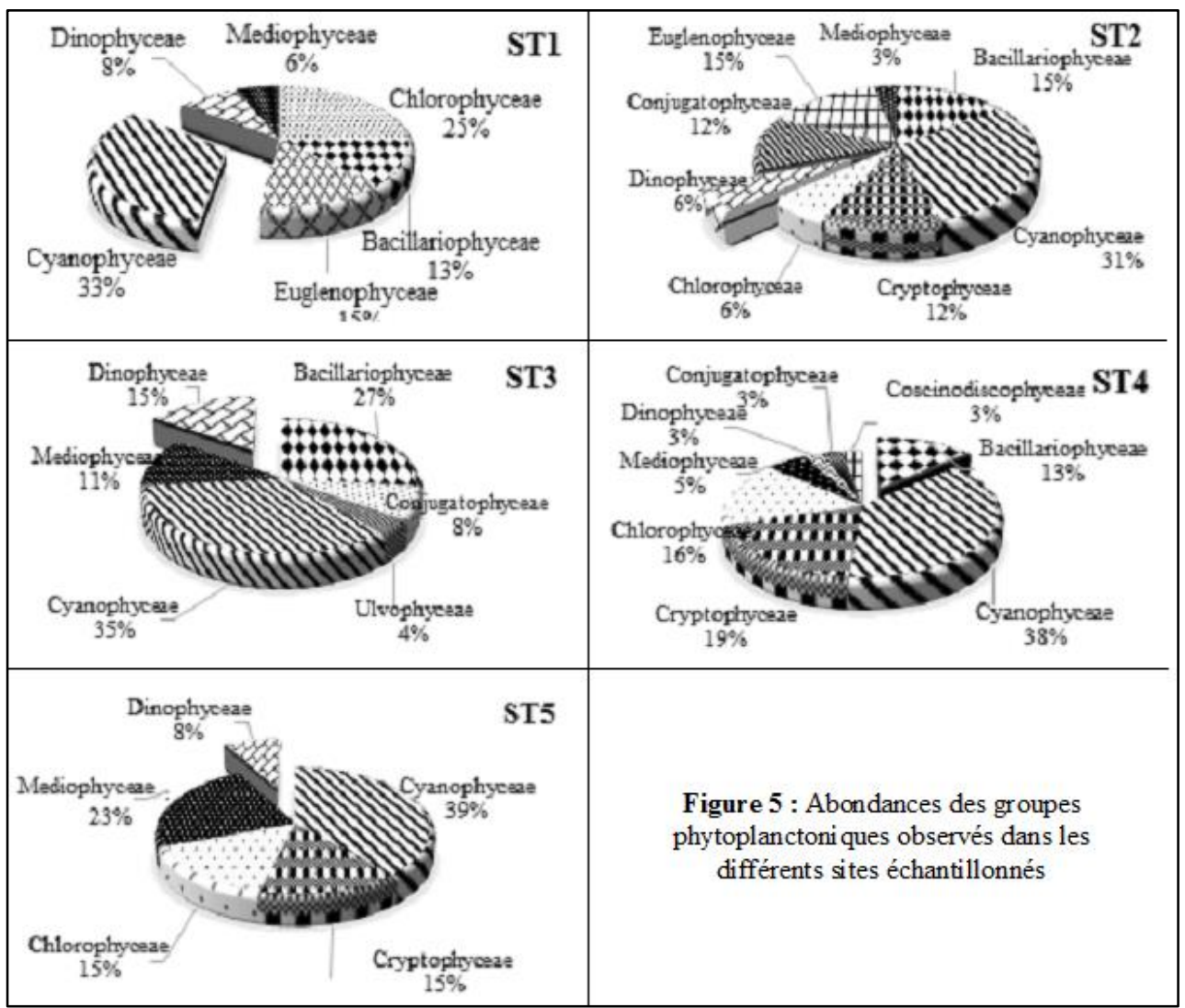

La deuxième station est caractérisée par l'abondance des Cyanophyceae (31\%), suivie par les Bascillariophyceae (15\%), Euglenophyceae (15\%), Cryptophyceae (12\%) et Conjugatophyceae (12\%), et $6 \%$ pour les Chlorophyceae et Dinophyceae et enfin, la plus basse fréquence (3\%) pour les Mediophyceae. La troisième station, caractérisée par la présence de six taxons est la plus diversifiée ; les Cyanophyceae et Bascillariophyceae représentent $62 \%$ du peuplement, les Dinophyceae : 15\%, Mediophyceae : $11 \%$, Conjugatophyceae : $8 \%$ et les Ulvophyceae, $4 \%$. Comparativement aux autres stations, la station 4 , présente une diversité importante après celle de la station 3. Les Cyanophyceae (38\%) sont les plus fréquentes puis viennent les 
Cryptophyteae (19\%), les Chlorophyceae (16\%), les Bascillariophyceae $(13 \%)$ et les Mediophyceae (5\%), alors que les autres classes viennent à la dernière position avec le même pourcentage (3\%). La dernière station c'est la moins diversifiée par sa position au niveau de la digue du barrage. Sa biodiversité est représentée par $39 \%$ de Cyanophyceae, $23 \%$ de Mediophyceae, les Cryptophyceae (15\%) sont aussi abondants que les Chlorophyceae (15\%) et les moins présents sont les Dinophyceae avec 8\%. Dans les cinq stations les Cyanophyceae est le groupe le plus fréquent. Ces dernières sont le composant phototrophique dominant dans beaucoup d'environnements d'eau douce où elles peuvent engendrer de sérieuses nuisances (Chorus et Bartram, 1999) à la fois écologiques et sanitaires vu que les eaux du barrage sont destinées, après traitement, à l'alimentation en eau potable des populations. Pendant la saison estivale, il est fréquent de constater d'importantes mortalités de poissons dans le lac dues à un déficit en oxygène et à l'impact d'efflorescences algales.

Tableau 2: Liste des taxons du phytoplancton des 5 stations du barrage Béni-Haroun

\begin{tabular}{|c|c|c|c|c|c|c|c|c|c|}
\hline Taxon & 1 & 2 & 34 & 5 & Taxon & 1 & 2 & 34 & 5 \\
\hline $\begin{array}{c}\text { Cyanophyceae } \\
\text { (Cyanobatéries) } \\
\text { Chroococcus sp } \\
\text { Microsystis sp }\end{array}$ & & $\begin{array}{l}+ \\
+ \\
\end{array}$ & & & $\begin{array}{c}\text { Bascillariophyceae } \\
\text { (diatomées) } \\
\text { Cymbella } s p \\
\text { Cyclotella } s p\end{array}$ & & + & ++ & $\begin{array}{l}+ \\
+ \\
\end{array}$ \\
\hline Arthrospira platensis & & + & & & Cyclotella meneghiniana & + & & & \\
\hline Oscillatoria spl & + & + & $+\quad+$ & + & Nitzschiaspl & + & + & ++ & + \\
\hline $\begin{array}{l}\text { Oscillatoria sp2 } \\
\text { Planktothrix sp }\end{array}$ & & & + & & $\begin{array}{l}\text { Nitzschia sp2 } \\
\text { Surirella sp }\end{array}$ & + & & + & \\
\hline Cylindrospermopsis sp & & & & + & Synedrasp & & + & & \\
\hline Anabena spiroide & & & $+\quad+$ & & Fragilaria sp & & + & & \\
\hline Anabaena spl & + & + & $+\quad+$ & & Diatoma spl & + & & + & \\
\hline $\begin{array}{l}\text { Anabaena sp2 } \\
\text { Anabaena sp3 }\end{array}$ & & & & & $\begin{array}{l}\text { Diatoma sp2 } \\
\text { Pinnularia sp }\end{array}$ & + & & ++ & \\
\hline Raphidiopsis $s p$ & & & + & + & Naviculaspl & & + & ++ & \\
\hline Snowella sp & + & & & & Naviculasp2 & & & ++ & \\
\hline Pseudoanabaenaspl & + & + & $+\quad+$ & + & Coscinodiscophyceae & & & & \\
\hline $\begin{array}{c}\text { Pseudoanabaena sp2 } \\
\text { Peridinium sp }\end{array}$ & + & + & $+\quad+$ & $\begin{array}{l}+ \\
+ \\
\end{array}$ & $\begin{array}{c}\text { Melosira varians } \\
\text { Melosira sp }\end{array}$ & & & $\begin{array}{r}+ \\
+\quad \\
\end{array}$ & \\
\hline Cryptophyceae & & & & & Chlorophyceaea & & & & \\
\hline Cryptomonas sp & + & + & $+\quad+$ & + & Chlamydomonas sp & + & + & ++ & + \\
\hline $\begin{array}{l}\text { Chroomonas } s p \\
\text { Euglenophyceae }\end{array}$ & & + & $+\quad+$ & & $\begin{array}{c}\text { Volvox sp } \\
\text { Gonium pectoale }\end{array}$ & $\begin{array}{l}+ \\
+ \\
\end{array}$ & & $\begin{array}{r}+ \\
+\end{array}$ & \\
\hline Euglena oxyuris & + & + & & & Zygnematophyceae & & & & \\
\hline Euglena sp & + & & & & Mougeotia sp & & + & + & \\
\hline Phacus sp & + & & & & Closterizom sp & & + & & \\
\hline $\begin{array}{l}\text { Ulvophyceae } \\
\text { Ulotrix zonata }\end{array}$ & & & + & & $\begin{array}{c}\text { Closterium aciculare } \\
\text { Staurastrum sp }\end{array}$ & & & $\begin{array}{l}+ \\
+\end{array}$ & \\
\hline
\end{tabular}


Pendant la période d'étude qui couvre un cycle annuel de janvier à décembre 2015, 44 genres ont été inventoriés dans les cinq stations d'étude (Tab.2). Ils appartiennent aux groupements phytoplanctoniques: Cyanophycées, Bacillariophycées (Diatomées), Chlorophycées, Zegnématophycées, Euglenophycées, Cryptophycées, Coscinodiscophycées, et les Ulvophycées. Les Cyanophyceae sont les plus dominants dans ces eaux eutrophisées indiquant une pollution organique (Mukherjee et al., 2010). Dans les 20 échantillons, le phytoplancton était dominé par les mêmes genres de cyanobactéries: Peridinium, Oscillatoria, Microsystis, Anabaena, et Pseudoanabaena, capables de produire des toxines. La contamination des milieux aquatiques par les cyanobactéries toxiques pose de sérieux risques pour les animaux domestiques, sauvages et pour la santé humaine (Brient et al., 2001). Les Chlorophycées sont souvent représentées par Chlamydomonas et Volvox avec l'apparition aboccasionnelle du Gonium dans les stations 1 et 4. Le genre Cyclotella, Navicula et Nitzchia ont été les plus représentatifs du groupe de Bascillariophycées. Les Euglenaphyceae représentées par Euglena et Phacus, sont bien abondants dans les stations 1et 2. Palmer (1969) a fait la première tentative majeure pour identifier et élaborer une liste de genres et d'espèces d'algues tolérantes à la pollution organique. Les genres comme Oscillatoria, Euglena, Chlamydomonas, Navicula et Nitzschia, sont décrites comme les plus tolérants aux eaux polluées.

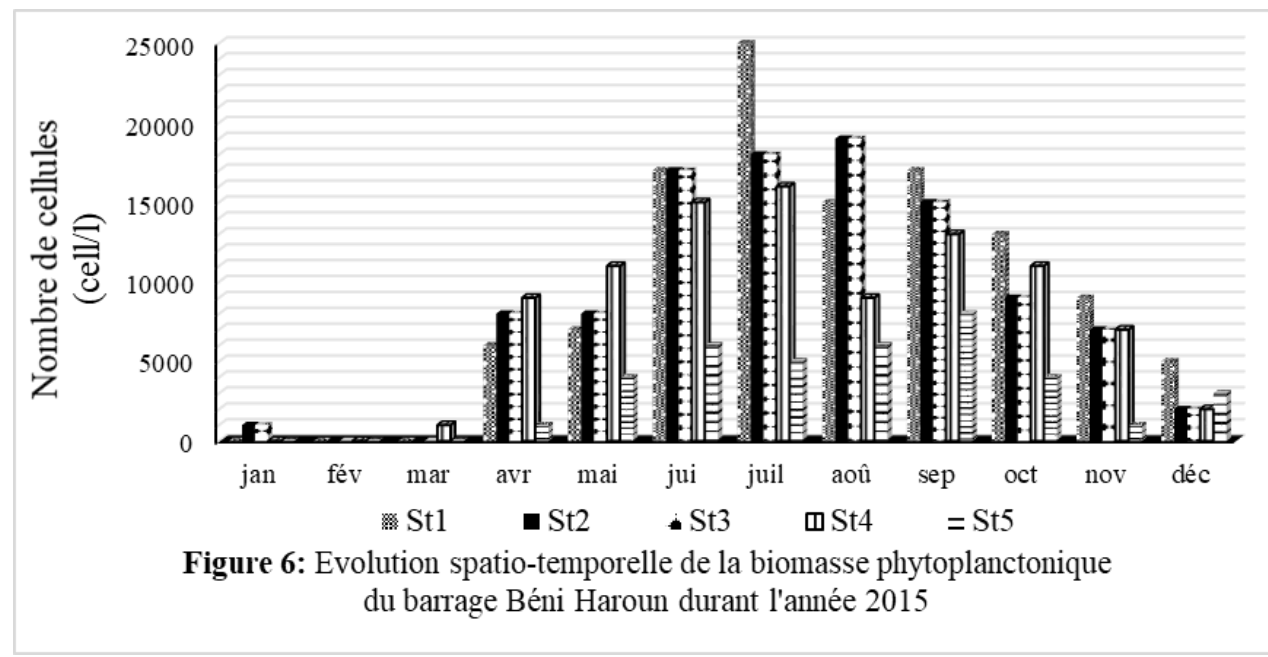

L'estimation mensuelle de la quantité de biomasse (Fig.6) a révélé un très faible développement moyen du phytoplancton, 2000 cell/1, durant le premier trimestre. Elle est représentée essentiellement par des diatomées et de Chlorophyceaee, c'est une situation de référence juste avant le début du développement printanier (température de l'air $<10^{\circ} \mathrm{C}$ et de l'eau $<20^{\circ} \mathrm{C}$ ). A cette période de l'année, l'activité phytoplanctonique est minimale, et la 
matière organique produite lors du cycle biologique précédent a été minéralisée. La quantité d'éléments nutritifs mesurée représente le stock dont pourra disposer le phytoplancton pour son développement printanier (Morin et al., 1991). Les mois d'avril et mai ont montré la première phase de croissance du phytoplancton avec une densité de 42000 cell/1, représenté essentiellement par des Cyanophyceae > Cryptophyceae, Bscillariophyceae, Chlorophyceae $>$ Mediophyceae. En plein été, un nombre maximum de cellules est enregistré en juin, juillet, août et même le mois de septembre, avec un pic le mois de juillet (25000 cell/1). A cette période de l'année, l'activité phytoplanctonique est maximale, largement dominée par les Cyanophyceae $>$ Chlorophyceae, Bascillariophyceae, Cryptophyceae $>$ Mediophyceae, Dinophyceae $>$ Euglenophyceae $>$ Zygnematophyceae. Une baisse régulière de la quantité est observée à la fin de septembre jusqu'à novembre c'est la fỉn de la stratification estivale, dominée essentiellement par les Cyanophyceae $>$ Diatomophyceae $>$ Chlorophyceae $>$ Dinophyceae et le reste avec une très faible participation. Au début de la saison hivernale marquée par la baisse de la température, le mois de décembre révèle un développement non négligeable du phytoplancton (3000 Cell/1), dominé par les Cyanophyceae lié probablement au climat régional. En effet, le climat méditerranéen chaud en été favorise la persistance des cyanobactéries dans les eaux douces eutrophes, qui peut commencer au printemps et persister jusqu'à décembre (Kormas et al., 2011 ; Sivonem et Jones, 1999). La dynamique des groupements algaux dans le temps est fortement liée aux variations des facteurs abiotiques dépendant des variations saisonnières. Des études dans les estuaires tropicaux suggèrent également que la saisonnalité influe sur la composition, la densité et la biomasse du phytoplancton, (Matos et al., 2011; Yin, 2002). Des fluctuations saisonnières de l'abondance ont été observées chez de nombreuses espèces, même dans un environnement dont la salinité varie peu (limnétique / oligohaline) (Chapman et al., 1998).

\section{Analyse statistique}

L'analyse de l'ANOVA (SPSS19), a montré des variations temporelles hautement à très hautement significatives pour la plupart des paramètres physico-chimiques et nutritifs. Cette dynamique est suivie par une variation très hautement significative $(p<0,01)$ de la quantité de biomasse phytoplanctonique (Tab.3). Cela explique bien que la structure de la communauté phytoplanctonique réagit fortement aux variables abiotiques, selon les préférences écologiques (Donadel et al., 2016 ; Bellinger et Sigee, 2010). La composition et l'abondance des espèces de plancton sont fonction des interactions avec les conditions environnementales, notamment la salinité, la température, la lumière et les éléments nutritifs (Mukherjee et al., 2010). Par contre, aucune variation significative dans l'espace n'a été enregistrée 
pour l'ensemble des paramètres biotiques et abiotiques traduisant ainsi une forte similarité entre les sites échantillonnés.

Tableau 3: Variations temporelles moyennes des paramètres biotiques et abiotiques des eaux du Barrage Béni-Haroun (ANOVA1, SPSS19). Les astérisques indiquent la probabilité du test ANOVA : *** : Très hautement significative, $* *$ : Hautement significative, ns : Non significative, $\mathrm{a}=12$ : Nombre de répétition, $\mathrm{St}$ : Station

\begin{tabular}{|c|c|c|c|c|c|}
\hline $\begin{array}{l}\text { Stations/ } \\
\text { Paramètres }\end{array}$ & Stl & St2 & St3 & St 4 & St5 \\
\hline $\mathrm{T}^{\circ} \mathrm{C}$ & $\begin{array}{c}23,2 \pm 4,3^{\mathrm{a}} \\
* * *\end{array}$ & $\begin{array}{c}21,7 \pm 5,5^{\mathrm{a}} \\
* * *\end{array}$ & $\begin{array}{c}23,4 \pm 7,4^{\mathrm{a}} \\
* * * *\end{array}$ & $\begin{array}{c}22,4 \pm 5,3^{\mathrm{a}} \\
* * *\end{array}$ & $\begin{array}{c}20,3 \pm 5,0^{\mathrm{a}} \\
* * * *\end{array}$ \\
\hline $\mathrm{pH}$ & $\begin{array}{c}7,9 \pm 0,8^{a} \\
* * * *\end{array}$ & $\begin{array}{c}7,9 \pm 0,5^{a} \\
* * * * *\end{array}$ & $\begin{array}{c}7,8 \pm 0,4^{a} \\
* * * *\end{array}$ & $\begin{array}{c}7,7 \pm 0,4^{a} \\
* * * *\end{array}$ & $\begin{array}{c}7,7 \pm 0,4^{a} \\
* * * *\end{array}$ \\
\hline $\mathrm{O} 2(\mathrm{mg} / \mathrm{l})$ & $\begin{array}{c}9,3 \pm 3,0^{a} \\
* * * *\end{array}$ & $\begin{array}{c}8,5 \pm 2,6^{a} \\
* * * *\end{array}$ & $\begin{array}{c}9,9 \pm 4,1^{a} \\
* * * *\end{array}$ & $\begin{array}{c}10,1 \pm 4,6^{a} \\
* * * *\end{array}$ & $\begin{array}{c}9,2 \pm 4,0^{a} \\
* * * *\end{array}$ \\
\hline $\begin{array}{l}\mathrm{CE}(\mu \mathrm{S} / \mathrm{cm}) \\
\mathrm{NO}_{3}^{-}(\mathrm{mg} /)\end{array}$ & $\begin{array}{c}1512 \pm 732^{\mathrm{a}} \\
\mathrm{ns} \\
10,4 \pm 2,4^{\mathrm{a}} \\
* *\end{array}$ & $\begin{array}{c}1239 \pm 123^{\mathrm{a}} \\
\mathrm{ns} \\
8,2 \pm 1,4^{\mathrm{a}} \\
* * \\
\end{array}$ & $\begin{array}{c}1194 \pm 74^{\mathrm{a}} \\
\mathrm{ns} \\
8,5 \pm 1,7^{\mathrm{a}} \\
* *\end{array}$ & $\begin{array}{c}1163 \pm 137^{\mathrm{a}} \\
\mathrm{ns} \\
10,4 \pm 1,5^{\mathrm{a}} \\
* *\end{array}$ & $\begin{array}{c}1166 \pm 88,7^{\mathrm{a}} \\
\text { ns } \\
8 \pm 1,4^{\mathrm{a}} \\
* * \\
\end{array}$ \\
\hline $\mathrm{NO}_{2}^{-}(\mathrm{mg} / \mathrm{l})$ & $\begin{array}{c}0,4 \pm 0,4^{\mathrm{a}} \\
* * * *\end{array}$ & $\begin{array}{c}0,34 \pm 0,38^{\mathrm{a}} \\
* * * *\end{array}$ & $\begin{array}{c}0,5 \pm 0,6^{\mathrm{a}} \\
* * * *\end{array}$ & $\begin{array}{c}0,5 \pm 0,5^{a} \\
* * * *\end{array}$ & $\begin{array}{c}0,4 \pm 0,4^{\mathrm{a}} \\
* * * *\end{array}$ \\
\hline $\mathrm{PO}_{4}^{-2}(\mathrm{mg} / \mathrm{l})$ & $\underset{* * *}{0,2 \pm 0,3^{\mathrm{a}}}$ & $\begin{array}{c}0,16 \pm 0,21^{\mathrm{a}} \\
* * * *\end{array}$ & $\begin{array}{c}0,1 \pm 0,2^{\mathrm{a}} \\
* * * *\end{array}$ & $\begin{array}{c}0,1 \pm 0,2^{\mathrm{a}} \\
* * * *\end{array}$ & $\begin{array}{c}0,1 \pm 0,2^{\mathrm{a}} \\
* * * *\end{array}$ \\
\hline $\mathrm{SO}_{4}^{-2}(\mathrm{mg} / 1)$ & $\begin{array}{c}328 \pm 173^{\mathrm{a}} \\
* * *\end{array}$ & $\begin{array}{c}288 \pm 120,8^{\mathrm{a}} \\
* * * *\end{array}$ & $\underset{* * *}{271 \pm 112,8^{a}}$ & $\begin{array}{c}274 \pm 115^{\mathrm{a}} \\
* * *\end{array}$ & $\underset{* * *}{281,6 \pm 103,7^{\mathrm{a}}}$ \\
\hline $\mathrm{Ca}^{++}(\mathrm{mg} / 1)$ & $\begin{array}{c}107 \pm 21^{\mathrm{a}} \\
* * * *\end{array}$ & $\begin{array}{c}94 \pm 16^{\mathrm{a}} \\
* * * *\end{array}$ & $\begin{array}{c}90 \pm 19,8^{\mathrm{a}} \\
* * * *\end{array}$ & $\begin{array}{c}92 \pm 20,8^{a} \\
* * * *\end{array}$ & $\begin{array}{c}85,9 \pm 14,7^{\mathrm{a}} \\
* * *\end{array}$ \\
\hline $\mathrm{Mg}^{++}(\mathrm{mg} / 1)$ & $\begin{array}{c}51 \pm 10,4^{\mathrm{a}} \\
* * * *\end{array}$ & $\underset{* * * *}{48,9 \pm 17,5^{\mathrm{a}}}$ & $\begin{array}{c}49,2 \pm 19,8^{a} \\
* * * *\end{array}$ & $\underset{* * *}{44,8 \pm 18,2^{a}}$ & $\underset{* * *}{53,9 \pm 16,9^{a}}$ \\
\hline $\mathrm{Cl}^{-}(\mathrm{mg} / \mathrm{l})$ & $\begin{array}{c}219 \pm 161^{\mathrm{s}} \\
\mathrm{ns}\end{array}$ & $\begin{array}{c}146,5 \pm 26,87^{\mathrm{a}} \\
\mathrm{ns}\end{array}$ & $\begin{array}{c}148,6 \pm 10,2^{\mathrm{a}} \\
\text { ns }\end{array}$ & $\begin{array}{c}146,6 \pm 24,8^{\mathrm{a}} \\
\mathrm{ns}\end{array}$ & $\begin{array}{c}139 \pm 18^{\mathrm{a}} \\
\mathrm{ns}\end{array}$ \\
\hline $\mathrm{Na}+(\mathrm{mg} / 1)$ & $\begin{array}{c}108 \pm 29^{a} \\
* * * *\end{array}$ & $\begin{array}{c}111 \pm 26,57^{\mathrm{a}} \\
* * * *\end{array}$ & $\begin{array}{c}114,5 \pm 21^{\mathrm{a}} \\
* * *\end{array}$ & $\begin{array}{c}106 \pm 27^{\mathrm{a}} \\
* * * *\end{array}$ & $\begin{array}{c}101 \pm 23^{\mathrm{a}} \\
* * * *\end{array}$ \\
\hline $\mathrm{K}^{+}(\mathrm{mg} / 1)$ & $\begin{array}{c}5,5 \pm 1,6^{a} \\
* * * *\end{array}$ & $\begin{array}{c}5 \pm 1,6^{a} \\
* * * *\end{array}$ & $\begin{array}{c}6 \pm 1,7^{a} \\
* * * *\end{array}$ & $\begin{array}{c}6,0 \pm 1,8^{a} \\
* * * *\end{array}$ & $\begin{array}{c}6,6 \pm 2^{a} \\
* * * *\end{array}$ \\
\hline $\begin{array}{l}\text { Biomasse } \\
(\text { Cell } / \mathrm{ml})\end{array}$ & $\begin{array}{c}9,5 \pm 8^{\mathrm{a}} \\
* * * *\end{array}$ & $\begin{array}{c}8,6 \pm 7^{\mathrm{a}} \\
* * * *\end{array}$ & $\begin{array}{c}8,6 \pm 7,13^{a} \\
* * * *\end{array}$ & $\begin{array}{c}7,8 \pm 5,8^{a} \\
* * *\end{array}$ & $\begin{array}{c}3 \pm 2,7^{\mathrm{a}} \\
* * * *\end{array}$ \\
\hline
\end{tabular}

Les communautés du phytoplancton, composante biotique majeure d'un écosystème aquatique sont grandement influencées par les différentes caractéristiques physico-chimiques de l'eau, selon la corrélation de Pearson. Cette dernière indique la force et la direction d'une relation linéaire entre les variables. D'après le résultat présenté dans le tableau 4, reflétant, la corrélation entre les paramètres abiotiques et la quantité de biomasse phytoplanctonique, on constate en générale que la quantité de biomasse est hautement corrélée positivement avec la température, le $\mathrm{pH}$ et les sulfates et négativement avec les phosphates, le magnésium, le sodium et le potassium, alors qu'avec le reste des paramètres, la corrélation n'est pas significative. Ce 
résultat est similaire à celui de (Donedel et al., 2016). La corrélation entre les éléments physico-chimiques, nutritifs et la quantité de biomasse algale est liée plus aux variations temporelles saisonnières. D'autres corrélations ont été bien observées entre les paramètres abiotiques eux même. D'après (Palmer, 1969), l'effet le plus important de la pollution organique dans un milieu aquatique est dû à l'enrichissement en substances nutritives et qu'il y a une corrélation claire entre la pollution organique et le développement du phytoplancton.

Tableau 4. Corrélations de Pearson entre la biomasse et les paramètres abiotiques $\left(*: \mathrm{P}<0,05 ;{ }^{* *}: \mathrm{P}<0,01\right)($ SPSS19)

\begin{tabular}{|c|c|c|c|c|c|c|c|c|c|c|c|c|c|}
\hline & $\mathrm{pH}$ & $\mathrm{O} 2$ & $\mathrm{CE}$ & $\mathrm{NO}^{\circ}$ & $\mathrm{NO}_{2}{ }^{-1}$ & $\mathrm{PO}_{4}{ }^{-2}$ & $\mathrm{SO}_{4}^{-2}$ & $\mathrm{Ca}^{+}$ & $\mathrm{Mg}^{+-}$ & a. & $\mathrm{Na}^{+}$ & $\mathrm{K}^{+}$ & Bicmasse \\
\hline $\mathrm{T}$ & $304^{\circ}$ & $348^{\circ}$ & & & &,$- 544^{\prime \prime}$ & $.724^{* *}$ & $0,999^{*}$ & $-430^{\circ}$ & &,$- 551^{*}$ &,$- 537^{*}$ & $827^{* *}$ \\
\hline $\mathrm{pH}$ & 1 & & & & & & $.555^{\circ *}$ & & $-325^{\circ}$ & & & &, $478^{* *}$ \\
\hline 02 & & 1 & & & $-260^{\circ}$ & $-292^{\circ}$ & & $393^{*}$ & $-482^{\circ *}$ & & & $\because 443^{* *}$ & \\
\hline $\mathrm{CE}$ & & & 1 & & & & & & & ,959** & & & \\
\hline $\mathrm{NO}_{3}{ }^{-}$ & & & & 1 & & & $-261^{\circ}$ & & & $.270^{\circ}$ & ,509" & & \\
\hline $\mathrm{NO}_{2}^{\circ}$ & & & & & 1 & & &,$- 439^{* *}$ & & & & & \\
\hline $\mathrm{PO}_{4}{ }^{-2}$ & & & & & & 1 &,$- 397^{* *}$ & &, $512^{*}$ & & $304^{\circ}$ & &,$- 445^{* *}$ \\
\hline $\mathrm{SO}_{4}^{-2}$ & & & & & & & 1 & & & &,$- 588^{* *}$ &,$- 272^{\circ}$ & $.768^{\circ *}$ \\
\hline $\mathrm{Ca}^{-}$ & & & & & & & & 1 & & & & $-273^{\circ}$ & \\
\hline $\mathrm{Mg}^{-}$ & & & & & & & & & 1 & & & $354^{* *}$ &,$- 382^{* *}$ \\
\hline $\mathrm{Cl}^{\circ}$ & & & & & & & & & & 1 & & & \\
\hline $\mathrm{Na}^{+}$ & & & & & & & & & & & 1 & $.492^{* *}$ &,$- 525^{*}$ \\
\hline $\mathrm{K}^{*}$ & & & & & & & & & & & & 1 &,$- 471^{* *}$ \\
\hline
\end{tabular}

Les résultats de l'ACP ( Fig.7) montrent que: Pour A, les deux premier axe expliquent $78 \%$ de variation totale disponible dans la matrice des donnés soumises à l'analyse (31.101\% axe1 et $46.925 \%$ axe 2$)$, dont l'analyse du composant principal a montré des corrélations positives et négatives entre la quantité de biomasse et les paramètres abiotique.

Pour B, les deux premiers axes expliquent $66.61 \%$ de variation total disponible dans la matrice des données soumise à l'analyse (24.946\% axe 1 et $41.663 \%$ axe 2), également l'ACP a montré des corrélations négatives entre l'abondance des groupes phytoplanctoniques et les éléments abiotique. 

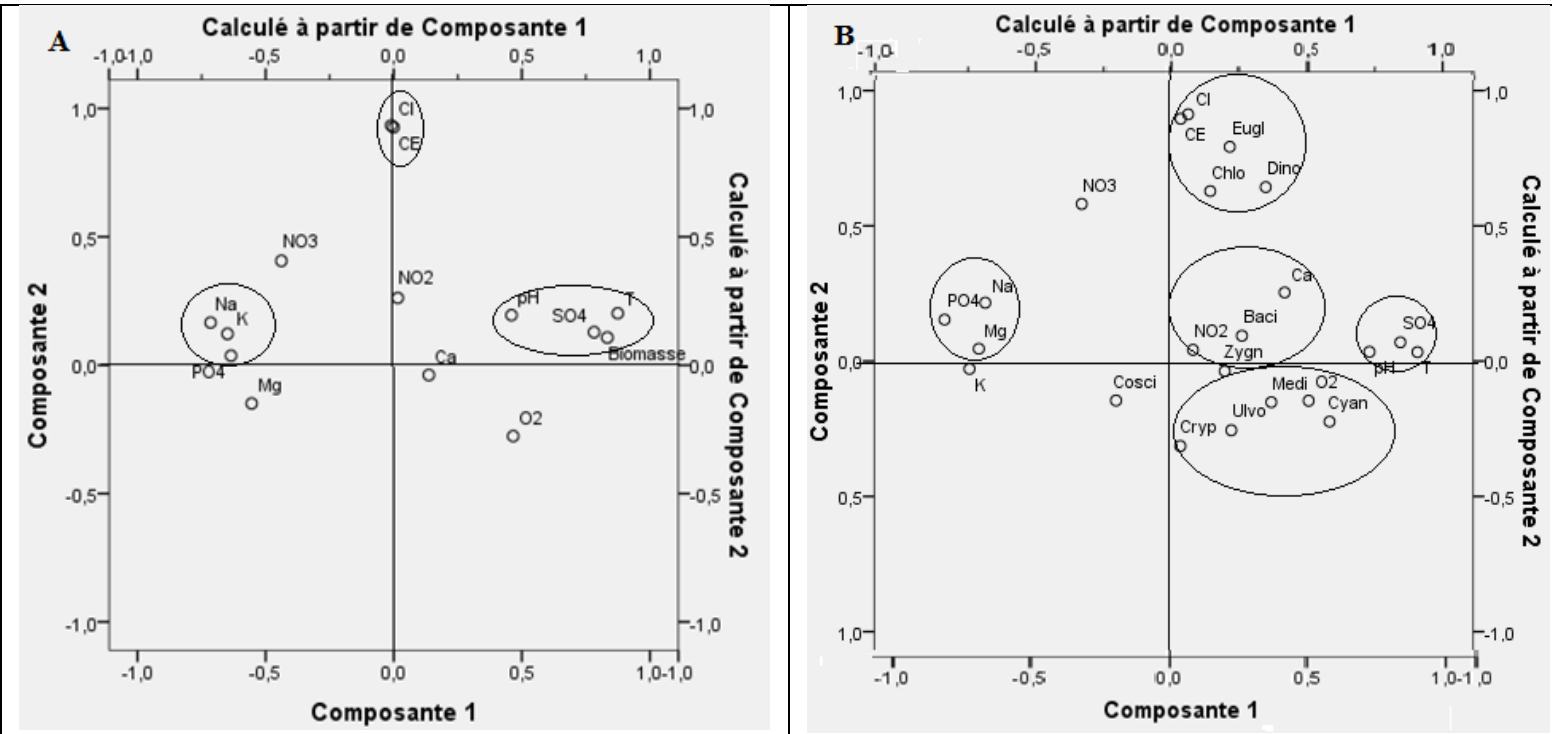

Figure 7: Analyse de composante principale expliquant la relation entre : (A) Variables environnementales et quantité de biomasse; (B) Variables environnementales et abondance des groupes phytoplanctonique.

Abréviations: Cryp-Cryptophyceae; Cyan-Cyanophyceae; Baci-Bacillariophyceae; Chlo-

Chlorophyceae; Eugl—Euglenophyceae; Cosci_Coscinodiscophyceae; Dino-Dinophyceae; MediMediophyceae; Ulvo-Ulvophyceae; Zygn-Zygnematophyceae.

\section{Conclusion}

Dans le présent travail, nous avons quantifié spatio-temporellement 13 paramètres physico-chimiques et nutritifs dans les eaux superficielles du barrage Béni-Haroun, au niveau de cinq sites répartis à la surface du plan d'eau. Le résultat montre des teneurs en éléments nutritifs qui ne sont pas encore alarmantes mais la situation risque de s'aggraver avec les différents apports de pollution qui ne cesse d'augmenter. Les éléments abiotiques ont montré des affinités positives et/ou négatives avec la quantité de biomasse phytoplanctonique, traduisant une légère pollution organique dans certains sites. L'inventaire phytoplanctonique a révélé la présence de 44 genres appartenant à huit groupes taxonomiques. Les Cyanophycées ont été le groupe le plus abondant dans la plupart des stations. La capacité d'envahissement des milieux aquatiques par les cyanobactéries est telle qu'elles entraînent parfois la quasi-disparition des autres micro-algues. Ceci est dû à leur capacité d'adaptation, et donc de compétition par rapport aux autres espèces phytoplanctoniques. Les eaux du barrage Béni-Haroun, le plus grand d'Algérie étant destinées à l'irrigation, à l'alimentation en eau des populations de cinq wilayas, et à la pêche, leurs utilisations pourraient avoir des conséquences sanitaires préoccupantes et une surveillance drastique de la qualité s'impose. 


\section{References:}

1. Abdallaoui, A. (1998). Contribution à l'étude du phosphore et des métaux lourds contenus dans les sédiments et de leur influence sur les phénomènes d'eutrophisation et de la pollution. Cas du bassin versant de l'oued Beht et de la retenue de barrage El Kansera. Thèse de doctorat. Université Moulay Ismail. Faculté des Sciences de Meknès, 209pp.

2. ABH. (1999). Aménagement des bassins hydrauliques. Constantine. Algérie

3. Afri, F., \& Pauwels, J.M. (1983).Facteurs physico-chimiques et présence de diatomées dans l'oued Rhumel, Mémoire de la Société d'Histoire Naturelle de l'Afrique du Nord.13:131-144.

4. Arrignon, J. (1976). Aménagement écologique et piscicole des eaux douces. Ed. Bords, Paris, 318 pp.

5. Badsil, H., Alil, O., Loudiki, M., \& Aamiril, A. (2012). American Journal of Human Ecology: Phytoplankton diversity and community composition along the salinity gradient of the Massa Estuary. 1: 58-64.

6. Balech, E. (1988). Los Dinoflagelados Del Atlantico Sud-occidental. Publ EspOcean1, 310pp

7. Benfetta, H., Ouadja, A., Achour, B., \& Remini, B. (2016). Larhyss Journal: Perte de capacité dans les barrages situes dans les zones arides et semi arides. Cas des barrages de Gargar, Bouhanifia, Ouizert et Foum el Gherza, 25 : 184-201.

8. Bourrelly, P. (1966). Les algues d'eau douce. Initiation à la systématique. Tome I : Les algues vertes. Eds. Boubée et Cie, Paris, $510 \mathrm{pp}$.

9. Bourrelly, P. (1968). Les algues d'eau douce. Initiation à la systématique. Tome II : Les algues jaunes et brunes, Chrysophycées, Phéophycées, Xanthophycées et Diatomées. Eds. Boubée et Cie, Paris, $438 \mathrm{pp}$.

10. Bourrelly, P. (1970). Les algues d'eau douce. Initiation à la systématique. Tome III: Les algues bleues et rouges. Les Eugléniens, Péridiniens et Cryptomonadines. Eds. Boubée et Cie, Paris, 512 pp.

11. Bouzid-Lagha, S., \& Djelita, B. (2012). Hydrological Sciences Journal: Etude du phénomène d'eutrophisation dans le Barrage de Hammam Boughrara (Wilaya de Tlemcen, Algérie). 57 (1) :186-201.

12. .Brient, L., Vézie, C., \& Bertru, G. (2001). Evaluation des efflorescences à cyanobactéries dans des eaux de cours d'eau et plans d'eau bretons. Rapport Université de Rennes I.U.M.R, Ecobio 6553. 35042 Rennes Cedex, 80pp.

13. Cambon, S. (2008). Services d'eau potable, de la logique d'offre à la maîtrise de la demande. Comparaison France-États- Unis. Thèse en 
Sciences et Techniques de l'Environnement, Ecole Nationale des Ponts et Chaussées, Paris, 429 pp.

14. Chapman, M.R., Funnell, B.M., \& Weaver, P.PE. (1998). Marine Micropaleontology: Isolation, extinction and migration within Late Pliocene populations of the planktonic foraminiferal lineage Globorotalia (Globoconella) in the North Atlantic., 203-222.

15. Chorus, I., \& Bartram, J. (1999). Toxic Cyanobacteria in Water. World Health Organization, 1st ed. E. and F.N. Spon, London and New York, 400pp.

16. Derradji, F., Bousnoubra, H., Kherici, N., Romeo, M., \& Caruba, R. (2007). Impact de la pollution organique sur la qualité des eaux superficielles dans le Nord-Est algérien. Sécheresse, 18 (1): 23-27.

17. Donadel, L., Cardoso, L. DE. S. \& Torgan, L.C. (2016). Plankton community dynamics in a subtropical lagoonal system and related factors. Anais da Academia Brasileira de Ciências , 88(1): 249-267

18. Drebes, G. (1976). Marine Phytoplankton: Auswahl der Helgolander Planktonalgen (Diatomeen, Peridineen). Ed. George Thiems, Stuttgart, $177 \mathrm{pp}$.

19. DTM, 2008. Direction des travaux maritimes. Mila. Algérie

20. El Ghachtoul, Y., Alaoui, M., \& Gabi, H. (2005). Eutrophisation des eaux des retenues des barrages Smir et Sehli (Maroc): Causes, conséquence et consignes de gestion. Revue des Sciences de l'Eau, 7589.

21. El Hachimi, O. (2012). Traitement des eaux usées par lagunage naturel en milieu désertique (Oasis de figuig) : Performances épuratoires et aspect phytoplanctonique. Thèse doctorat. Université Mohammed Premier. Faculté des Sciences Oujda, 140pp.

22. Ellinger, E.G., \& Sigee, D.C. (2010). Fresh water Algae: identification and use as bioindicators. Chichester: J Wiley \& Sons, 271pp.

23. Findlay, D.L. \& Kling, H.J. (1990). Protocols for measuring biodiversity: Phytoplankton in Fresh water. Department of Fisheries and Oceans. Fresh water Institute 501 University. Crescent Winnipeg, Manitoba. N6, 19pp

24. Fouad, S., Hajjami, K., Cohen, N., \& Chlaida, M. (2014). Afrique science: Qualité physico-chimique et contamination métallique des eaux de l'Oued Hassar : impacts des eaux usées de la localité de Mediouna (Périurbain de Casablanca, Maroc), 10(1) : 91-102.

25. Germain, H. (1981). Flore des Diatomées. Eaux douces et saumâtres. Eds. Boubée et Cie, Paris, 443pp.

26. Harrat, N., \& Achour, S. (2006). Qualité des eaux de surface de l'Est algérien et leur réactivité vis-à-vis du chlore ; Mémoire de Magister en géologie, 62pp. 
27. Harrat, N,. \& Achour, S. (2010). Larhyss Journal : Pollution physicochimique des eaux de barrage de la région d'El Tarf. Impact sur la chloration, 8: 47-54.

28. Joradp. (2011). Décret excécutif $n^{\circ} 11-219$ du 12 juin 2011 fixant les objectifs de qualité des eaux superficielles et souterraines destinées à l'alimentation en eau des populations. Joradp n³4 du 19 juin 2011.

29. Kormas, K. Ar., Gkelis S, Vardaka, El., \& Moustaka-Gouni, M.(2011). Limnologica: Morphological and molecular analysis of bloomforming Cyanobacteria in two eutrophic, shallow Mediterranean lakes, 41: 167-173

30. Krammer, K., \& Lange-Bertalot, H. (1986). Naviculaceae. Süßwasserflora von Mitteleuropa 2/1. Gustav Fischer Verlag. Stuttgart, 876pp.

31. Krammer, K., \& Lange-Bertalot, H. (1988). Bacillariaceae, Epithemiaceae, Surirellaceae. Süßwasserflora von Mitteleuropa 2/2. Gustav Fischer Verlag. Stuttgart, 596pp.

32. Krammer, K., \& Lange-Bertalot, H. (1991a). Centrales, Fragilariaceae, Eunotiaceae. Süßwasserflora von Mitteleuropa 2/3. Gustav Fischer Verlag. Stuttgart, 576pp.

33. Krammer, K., \& Lange-Bertalot, H. (1991b). Achnanthaceae. Süßwasserflora von Mitteleuropa 2/4. Gustav Fischer Verlag. Stuttgart, 437 pp.

34. Lavoie, I., Hailton, PB., Campeau, S., Grenier, M., \& Dillon, PJ., (2008). Guide d'identification des diatomées des rivières de l'est de Canada. Presse de l'université du Québec. Imprimé au Canada, 241pp.

35. Matos, J.B., Sodré, D.K.L., Da Costa, KG., Pereira, LCC., \& Da Costa, RM. (2011). Spatial and temporal variation in the composition and biomass of phytoplankton in an Amazonian estuary. J Coastal Res SI, 64: 1525-1529.

36. Mebarki, A., Benabbas, Ci., \& Grec, F. (2008). Le système « Béni Haroun » (oued Kebir- Rhumel, Algérie): Aménagements hydrauliques et contraintes morpho-géologiques, 37-51.

37. Morin, P., LE Corre, P., Marty, Y., \& L'Helguen, S. (1991). Oceanologica Act : Evolution printanière des éléments nutritifs et du phytoplancton sur le plateau continental armoricain Europe de NordOuest.3 : 263-280

38. Mukherjee, B., Nivedita, M., \& Mukherjee, D. (2010). Journal of Environmental Biology: Plankton diversity and dynamics in a polluted eutrophic lake, Ranchi. 31(5): 827-839.

39. OMS, (2004). Guidelines for drinking-water quality, Vol. 1, Recommendations, 3rd Ed., World Health Organization, Geneva.

40. Palmer, C.M. (1969). Journal of Phycology: A Composite rating of 
Algae Toleraing Organic Pollution., 5: 75-82.

41. Sivonen, K., \& Jones G. (1999). Edited by Ingrid Chorus and Jamie Bartram: Toxic Cyanobacteria in Water: A guide to their public health consequences, monitoring and management. 55-124.

42. Smruti, S. (2016). India International Journal of Science Technology \& Engineering: A Review of Phytoplankton Ecology in Freshwater Rivers and Lakes of 3: 19-21.

43. Rodier, J., Bernard, L., Nicole, M., \& coll, (2009). L'analyse de l'eau. $9^{\mathrm{e}}$ édition Dunod Paris, 1959, 1579 pp.

44. Yin, K. (2002). Marine Ecology Progress Series: Monsoonal influence on seasonal variations in nutrients and phytoplankton biomass in coastal waters of Hong Kong in the vicinity of the Pearl River estuary. 245: 111-122. 